Reprod. Nutr. Dévelop., 1980, 20 (4 B), 1173-1179.

\title{
Evacuation gastrique des aliments et de l'éthanol administrés seuls ou conjointement
}

par B. LE BOURHIS, Y. MÉNORET

avec la collaboration technique de J. P. TERRANOVA

Institut de Recherches Appliquées aux Boissons, 120, avenue Foch, 94015 Créfeil (France).

Summary. Gastric emptying of food and ethanol given alone or together.

In rats as in man, the simultaneous intake of carbohydrates (glucose, sucrose, fructose at $1 \mathrm{~g} / \mathrm{kg})$, soya proteins $(1 \mathrm{~g} / \mathrm{kg}$ ) or lab chow $(3.5 \mathrm{~g} / \mathrm{kg})$ reduced blood alcohol levels after the intake of $1 \mathrm{~g} / \mathrm{kg}$ of alcohol.

This reduction was greater with protein than with carbohydrates, and was apparently not due to an increase of ethanol metabolism but to a delay in the gastric emptying of alcohol.

On the other hand, we observed that the latter was 4 to 8 times faster than that of food.

\section{Introduction.}

De très nombreuses observations montrent que l'alcool absorbé au cours d'un repas provoque une alcoolémie moindre que s'il est ingéré à jeun. La majorité des chercheurs ont reconnu que les sucres et en particulier le fructose réduisaient l'alcoolémie. Par contre, les effets des protéines et des acides aminés sur l'alcoolémie ont été beaucoup moins étudiés. Les explications apportées à ces phénomènes sont nuancées et même parfois contradictoires. Certains auteurs estiment qu'il s'agit d'une accélération du métabolisme de l'alcool, d'autres pensent au contraire que la réduction de l'alcoolémie est due à un retard apporté par les aliments à la diffusion de l'alcool dans l'organisme.

Dans des études antérieures, nous avons vérifié chez l'homme l'effet hypoalcoolémiant de sucres (glucose, fructose, sucre de maïs isomérisé), d'aliments à haute teneur en protéines végétales ou animales (Le Bourhis et Aufrère, 1976) ef de tanins extraits du raisin (résultats non publiés). Dans une nouvelle série d'expériences réalisées sur le rat, nous avons cherché à reproduire les effets observés chez l'homme, puis à les expliquer en étudiant simultanément les cinétiques d'évacuation gastrique des aliments et de l'alcool, ainsi que la vitesse de dégradation de l'alcool. 


\section{Matériel et méthodes.}

Animaux : rats Wistar mâles de $250 \mathrm{~g}$ environ, à jeun depuis 18 heures.

Dosages ef mesures : alcool dans le sang : microméthode enzymatique à l'ADH. Alcool dans l'estomac : on prélève l'estomac que l'on distille en présence d'eau, puis on dose l'alcool dans le distillat.

Alcool dans l'organisme entier : l'animal est sacrifié puis découpé en une vingtaine de morceaux. Il est ensuite broyé au mixer (Turmix). Dans le bol de l'appareil on introduit le rat, $200 \mathrm{ml}$ d'eau distillée et environ $100 \mathrm{mg}$ de FNa. Le broyage est réalisé en chambre froide $\left(-25^{\circ} \mathrm{C}\right)$ et interrompu fréquemment afin d'éviter l'échauffement du broyat. On distille et on dose l'alcool dans le distillat (taux de recouvrement de l'alcool $\geqslant 96$ p. 100). Provende dans l'estomac: on prélève l'estomac, on récupère la provende que l'on dessèche à poids constant.

\section{Protocoles expérimentaux.}

a) Ełude des effets des aliments sur l'alcoolémie et la vitesse d'évacuation gastrique. Dans une première expérience, nous avons déterminé la valeur de l'alcoolémie et la quantité d'alcool restant dans l'estomac $30 \mathrm{~min}$ après le traitement. Les rats étaient répartis en 6 groupes de 6 ou 8 et recevaient par intubation œesophagienne $1 \mathrm{~g} / \mathrm{kg}$ d'alcool (éthanol à 200 $\mathrm{GL}$ ) et immédiatement après, soit une solution de sucre (saccharose, fructose, glucose), soit une suspension de protéines (Promine D), suivant les indications portées sur le fableau 1 et correspondant dans chaque cas à une absorption de $1 \mathrm{~g} / \mathrm{kg}$ de sucre ou de protéines. Les animaux étaient sacrifiés $30 \mathrm{~min}$ après le traitement et on déterminait leur alcoolémie et la quantité d'alcool contenue dans leur tube digestif. Dans une deuxième expérience, nous avons étudié l'évolution de l'alcoolémie et celle de la teneur en alcool de l'estomac chez des rats ayant reçu de l'alcool seul ou de l'alcool accompagné de provende, Les animaux recevaient par sonde œsophagienne $2,5 \mathrm{ml}$ d'eau (témoins) ou $2,5 \mathrm{ml}$ de suspension de provende (biscuit Extralabo) correspondant à $3,5 \mathrm{~g} / \mathrm{kg}$ de nourriture sèche (traités), immédiatement suivis de $1 \mathrm{~g} / \mathrm{kg}$ d'alcool. Ils étaient sacrifiés : 10, 20, 30, 45, 60, 90,120, $180 \mathrm{~min}$ après le traitement et l'on déterminait leur alcoolémie et la teneur en alcool de leur tube digestif.

b) Effet de l'alcool sur la vitesse d'évacuation gastrique des aliments. - Les rats recevaient par sonde œsophagienne $1 \mathrm{~g} / \mathrm{kg}$ d'alcool immédiatement suivi de $2,5 \mathrm{ml}$ d'eau ou de $2,5 \mathrm{ml}$ d'une suspension de provende correspondant à $3,5 \mathrm{~g} / \mathrm{kg}$. Ils étaient sacrifiés $30,60,120,180$ ou 240 min plus tard et on déterminait la quantité de provende restant dans l'estomac.

c) Effet des aliments sur la vitesse d'oxydation de l'alcool. - Les rats recevaient par voie orale $2,5 \mathrm{ml}$ d'eau, de solution de sucre ou de suspension de protéines correspondant à $1 \mathrm{~g} / \mathrm{kg}$ de sucre ou de protéines. Trente minutes plus tard, tous les animaux recevaient $1 \mathrm{~g} / \mathrm{kg}$ d'alcool par voie IP. Deux heures plus tard, on déterminait l'alcool restant dans l'organisme et on en déduisait la vitesse de dégradation de l'alcool (coefficient d'éthyloxydation : CEO).

$$
\mathrm{CEO}=\frac{\text { alcool administré }(\mathrm{mg})-\text { alcool restant }(\mathrm{mg})}{\text { poids de l'animal }(\mathrm{kg}) \times \operatorname{temps}(2 \mathrm{~h})} .
$$




\section{Résultats.}

a) Effet des aliments sur l'alcoolémie ef la vifesse d'évacuation gastrique. - Les résultats de la première expérience sont rassemblés dans le tableau 1 . On constate que l'alcoolémie des animaux est toujours plus faible si l'ingestion d'alcool a été accompagnée de celle d'un sucre ou de protéines que si elle a été accompagnée d'eau. Tous les résultats obtenus sont statistiquement significatifs $(P<0,01)$. Cetfe réduction d'alcoolémie varie de 40 à 80 p. 100. Parallèlement, il apparaît que la teneur en alcool du tube digestif est plus importante chez les animaux traités que chez les témoins de 240 à 430 p. 100 selon les cas $(P<0,05$ ou 0,01). La Promine $D$ semble

\section{TABLEAU 1}

Effet des sucres ef des protéines sur la valeur de l'alcoolémie ef la quantité d'alcool restant dans l'estomac 30 min après ingestion de $1 \mathrm{~g} / \mathrm{kg}$ de sucres ou de protéines

\begin{tabular}{|c|c|c|c|c|c|c|c|c|}
\hline \multirow{2}{*}{$\begin{array}{c}\text { Substance } \\
\text { administrée } \\
\text { en plus } \\
\text { de l'alcool }\end{array}$} & \multirow{2}{*}{$\begin{array}{c}\text { Nombre } \\
\text { d'animaux } \\
\text { utilisés }\end{array}$} & \multirow{2}{*}{$\begin{array}{l}\text { Concentration } \\
\text { de la solution } \\
\text { ou suspension } \\
\text { en } \mathrm{mg} / \mathrm{ml}\end{array}$} & \multirow{2}{*}{$\begin{array}{l}\text { Volume } \\
\text { administré } \\
\text { en mg/rat }\end{array}$} & \multirow{2}{*}{$\begin{array}{c}\text { Dose } \\
\text { administrée } \\
\text { en } \mathrm{mg} / \mathrm{rat}\end{array}$} & \multicolumn{2}{|c|}{\begin{tabular}{|} 
Substances \\
admi- \\
nistrées \\
en $\mathrm{mg} / \mathrm{kg}$
\end{tabular}} & \multirow{2}{*}{$\begin{array}{l}\text { Alcoolémie } \\
\text { à la } 30 \text { e min } \\
\text { en } g / l=S m\end{array}$} & \multirow{2}{*}{$\begin{array}{c}\text { Alcool } \\
\text { restant dans } \\
\text { l'estomac } \\
\text { d la } 30^{\mathrm{e}} \mathrm{min} \\
\text { en } \mathrm{mg} \pm \mathrm{Sm}\end{array}$} \\
\hline & & & & & $\begin{array}{l}\text { glu- } \\
\text { cides }\end{array}$ & $\begin{array}{l}\text { pro- } \\
\text { tides }\end{array}$ & & \\
\hline $\begin{array}{l}\text { Eau ...... } \\
\text { Saccharose } \\
\text { Glucose ... } \\
\text { Fructose .. } \\
\text { Promine D. }\end{array}$ & $\begin{array}{l}6 \\
6 \\
6 \\
6 \\
8\end{array}$ & $\begin{array}{l}\overline{167} \\
167 \\
167 \\
105\end{array}$ & $\begin{array}{l}1,5 \\
1,5 \\
1,5 \\
1,5 \\
2,5\end{array}$ & $\begin{array}{l}-\overline{250} \\
250 \\
250 \\
262\end{array}$ & $\begin{array}{l}1 \overline{1} 000 \\
1000 \\
1000 \\
-\end{array}$ & $\begin{array}{l}- \\
\overline{-} \\
1 \overline{0}\end{array}$ & $\mid \begin{array}{l}1,16 \pm 0,07 \\
0,58 \pm 0,05 * * \\
0,70 \pm 0,08 * * \\
0,66 \pm 0,05 * * \\
0,28 \pm 0,03 * *\end{array}$ & $\begin{array}{l}14,8 \pm 2,9 \\
37,0 \pm 8,5 * \\
34,7 \pm 6,8 * * \\
37,5 \pm 4,3 * * \\
41,6 \pm 3,5 * *\end{array}$ \\
\hline
\end{tabular}

Test $F * P<0,05 ; * * P<0,01$.

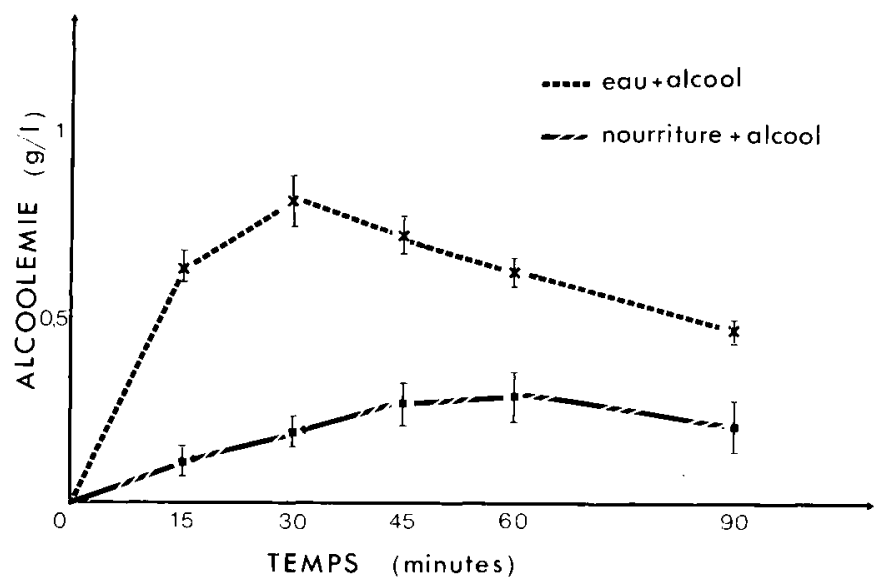

FIG. 1. - Evolution de l'alcoolémie chez le rat oprès ingestion de $1 \mathrm{~g}$ 'kg d'alcool associée ou non à celle de provende. 
plus efficace que les sucres. Les résultats de la deuxième expérience sont représentés par les figures 1 et 2 . Ils confirment et précisent ceux de la première. Remarquons tout d'abord qu'expérimentalement on ne trouve dans l'estomac guère plus de 90 p. 100 de l'alcool administré. Ce déficit est dû pour une part au fait qu'au temps «zéro » le délai séparant le gavage et l'ablation de l'estomac n'est pas nul, pour une autre part au rendement du dosage d'alcool dans l'estomac qui n'est que de l'ordre de 96 p. 100. La figure 1 montre que sous l'influence de la provende, la courbe d'alcoolémie est profondément modifiée. Le classique pic qui apparaît normalement $30 \mathrm{~min}$ après l'ingestion d'alcool est considérablement aplati $(0,3 \mathrm{~g} / /$ au lieu de $0,8 \mathrm{~g} / \mathrm{l})$ et retardé (60 min après l'ingestion au lieu de 30$)$. Par ailleurs, la vitesse d'évacuation gastrique de l'alcool est, elle aussi, profondément modifiée sous l'influence de la provende (fig. 2). Chez les témoins n'ayant reçu que l'alcool, l'évacuation gastrique est pratiquement terminée après $30 \mathrm{~min}$. Chez les traités, elle est très retardée et ne commence que $30 \mathrm{~min}$ après l'administration d'alcool et de provende. A partir de ce moment, l'évacuation de l'alcool se fait à la même vitesse pour les deux groupes d'animaux, autrement dit l'ingestion de provende simultanément à l'alcool ne ralentif pas l'évacuation gastrique mais la refarde de façon importante.

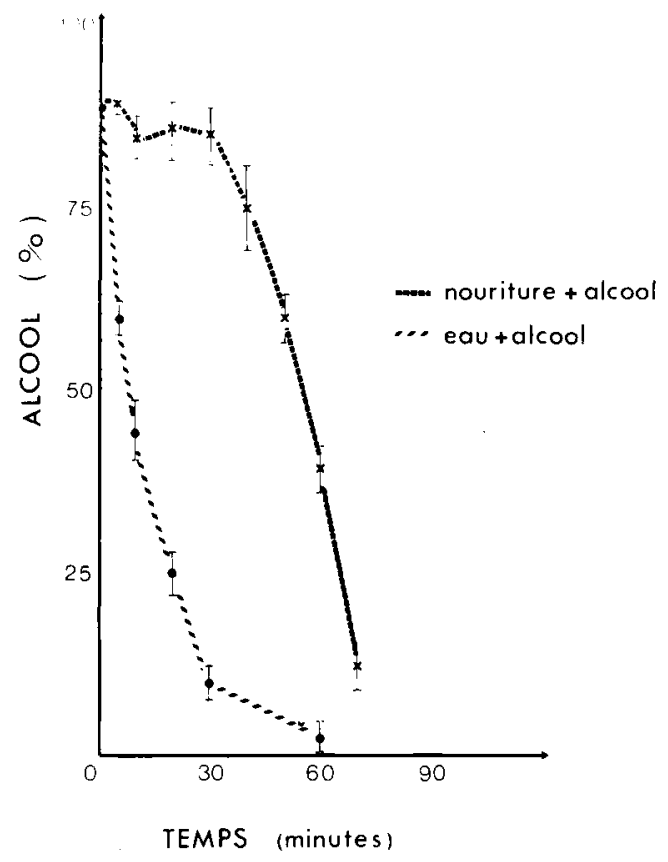

FIG. 2. - Cinétique de l'évacuation gastrique de l'alcool chez le rat oprès ingestion de $1 \mathrm{~kg} / \mathrm{g}$ d'alcool associé ou non à $3,5 \mathrm{~g} / \mathrm{kg}$ de provende.

b) Effet de l'alcool sur la vifesse d'évacuation gastrique des aliments. - La figure 3 représente les résultats obtenus. On constate que l'alcool commence par accélérer l'évacuation gastrique des aliments pendant la première demi-heure $(P<0,001)$, puis la ralentit entre la $2^{e}$ et la $3^{\mathrm{e}}$ heure $(\mathrm{P}<0,001$ ef $\mathrm{P}<0,05$ respectivement) pour 
aboutir finalement à la même déplétion $4 \mathrm{~h}$ après l'administration d'alcool et de provende.

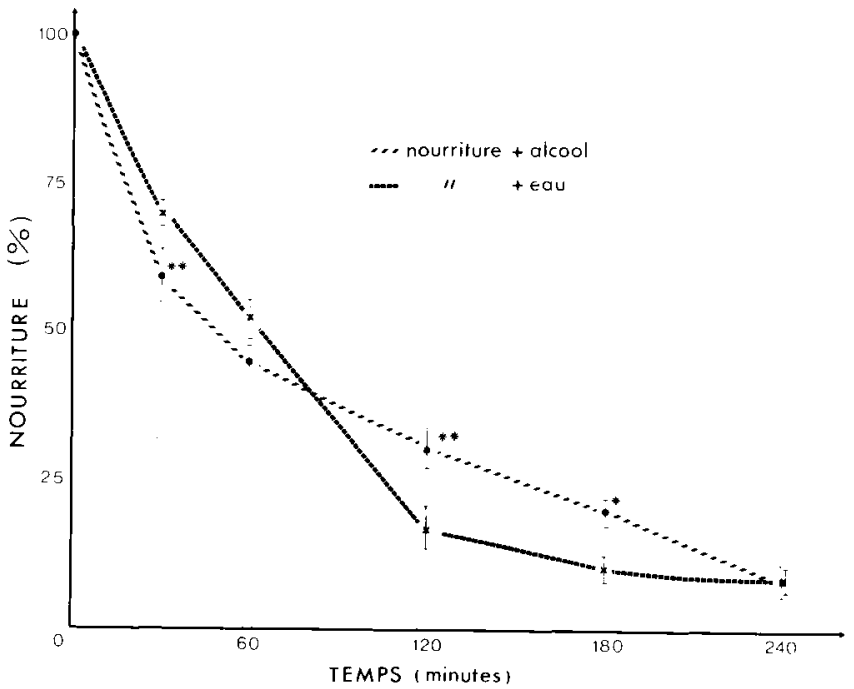

FIG. 3. - Cinétique d'évacuation gastrique de la nourriture chez le rat oprès ingestion de $3,5 \mathrm{~g} / \mathrm{kg}$ de provende associée ou non ò $1 \mathrm{~g} / \mathrm{kg}$ d'alcool.

c) Effet des aliments sur la vitesse d'oxydation de l'alcool. - Les résultats obtenus sont rassemblés dans le tableau 2. On constate que ni les sucres, ni les protéines ne modifient de façon significative la valeur du CEO.

TABLEAU 2

\begin{tabular}{|c|c|c|}
\hline $\begin{array}{l}\text { Substance administrée } \\
\text { simultanément à l'alcool }\end{array}$ & $\begin{array}{c}\text { Dose administrée } \\
\mathrm{mg} / \mathrm{rat}\end{array}$ & $\begin{array}{c}\mathrm{CEO} \\
\mathrm{mg} / \mathrm{kg} / \mathrm{h} \pm \mathrm{Sm}\end{array}$ \\
\hline $\begin{array}{l}\text { Eau } \ldots \ldots \ldots \ldots \ldots \ldots \\
\text { Saccharose } \ldots \ldots \ldots \ldots \ldots \\
\text { Fructose } \ldots \ldots \ldots \ldots \ldots \ldots \\
\text { Promine } D \ldots \ldots \ldots \ldots \\
\text { Aliment protéiné } \ldots \ldots \ldots\end{array}$ & $\begin{array}{l}\overline{250} \\
250 \\
262 \\
712\end{array}$ & $\begin{array}{l}351 \pm 9 \\
361 \pm 14 \text { (NS) } \\
334 \pm 8 \text { (NS) } \\
362 \pm 8 \text { (NS) } \\
358 \pm 5 \text { (NS) }\end{array}$ \\
\hline
\end{tabular}

Test $F: N S P>0,05$.

\section{Discussion et conclusions.}

Nos résultats confirment donc que l'ingestion d'aliments conjointement à de l'alcool réduit l'alcoolémie. Les protéines sont plus efficaces que les sucres, ce qui confirme les résultats de Jarrige (1970 $a$ et $b$ ). En comparant les figures 2 et 3, on constate que chez les animaux recevant l'alcool et la provende, l'élimination de la nourriture ef celle de l'alcool ne se font pas à la même vifesse. Ainsi, 30 min après 
l'intubation, tout l'alcool administré est encore dans l'estomac (fig. 2), alors que 30 p. 100 de la provende ont déjà été évacués (fig. 3) ; 70 min après l'intubation, il ne reste dans l'estomac que 15 p. 100 de l'alcool administré (fig. 2) mais encore 40 p. 100 de la provende (fig. 3). Ce résultat esł d'ailleurs conforme à ce que l'on observe couramment dans les expériences d'alcoolémie provoquée. En effet, on sait que la disparition de l'alcool de l'estomac correspond au maximum d'alcoolémie et l'on constate que le maximum survient 20 à 60 min après l'absorption d'alcool selon qu'elle a lieu à jeun ou au contraire accompagnée d'un repas. Ceci ne peut s'expliquer que si l'on admet que l'alcool est éliminé avec la phase liquide 4 à 8 fois plus rapidement que la phase solide.

D'après nos résultats, la réduction de l'alcoolémie ne serait pas due à une augmentation de la vitesse de dégradation de l'alcool, ni dans le cas des sucres, ni dans celui des protéines. Ce point particulier est d'ailleurs toujours l'objet d'une certaine controverse. Pour certains auteurs, le fructose accélèrerait le métabolisme de l'éthanol (Stuhlfault ef Neumaier, 1954 ; Lundquist et Wolthers, 1958 ; Lowenstein ef al., 1970), pour d'autres ni les protéines ni les sucres ne modifient le CEO (Le Breton, 1934 ; Dontcheff, 1937 ; Dundee, Bovill et Isaac, 1971). En ce qui concerne l'interaction de l'alcool et des aliments sur l'évacuation gastrique, on constate que l'alcool tend à ralentir faiblement l'évacuation des aliments, mais qu'inversement les sucres et plus encore les protéines ralentissent l'évacuation gastrique de l'alcool. Ce ralentissement apparaît d'ailleurs dans notre étude comme la seule cause de la réduction de l'alcoolémie. Cet effet avait été rapporté à propos du glycocolle par Haggard et Greenberg (1940) mais à notre connaissance n'avait pas encore été étendu aux protéines. Par ailleurs, Clark, Hughes et Letley (1973) ayant étudié chez l'homme les courbes d'alcoolémie consécutive à l'absorption d'alcool associé à divers sucres avaient estimé, sans pouvoir le démontrer, que les modifications observées étaient dues à une alternation de l'absorption de l'éthanol à partir de l'appareil gastrointestinal. L'effet retardateur des sucres, des protéines et de la provende sur l'évacuation gastrique peut sans doute être expliqué par la mise en jeu des récepteurs duodénaux multiples, pH sensibles, osmosensibles et liposensibles qui viendraient déclencher les mécanismes rétro-inhibiteurs de l'évacuation gastrique.

\section{Journées Ingestion-Digestion-Absorption de l'Association française de Nutrition, Poris, 15-16 novembre 1979.}

\section{Références}

CLARK E. R., HUGHES I. E., LETLEY E., 1973. The effect of oral administration of various sugars on blood ethanol concentrations in man. J. Pharm. Pharmac., 25, 319-322.

DONTCHEFF L., 1937. Influence des divers types d'aliments sur la vitesse d'oxydation de l'alcool éthylique chez l'homéotherme inanitié (rat blanc), C. R. Soc. Biol., 126, 465-467.

DUNDEE J. W., BOVILL J., ISAAC M., 1971. Failure to demonstrate an increased removal of alcohol from the blood stream by fructose, Med. Sci. Law, 11, 146-148.

HAGGARD H. W., GREENBERG L. A., 1940. Studies on the absorption, distribution and elimination of alcohol. IV. The influence of glycocol upon the absorption of alcohol. J. Pharm. exp. Ther., 68, $482-493$.

JARRIGE P., 1970a. Etude de l'influence de la nature des aliments ingérés sur l'alcoolémie chez l'homme. Bull. Acad. nat. Med., 154, 340-344. 
JARRIGE P., 1970b. Etude de l'influence de divers sucres sur l'alcoolémie chez l'homme. Bull. Acad. nat. Med., 154, 344-350.

LE BRETON E., 1934. Influence de la nature de l'aliment brôlé sur la vitesse d'oxydation de l'alcool dans l'organisme. Cas des protides. C. R. Soc. Biol., 117, 709-712.

LE BOURHIS B, AUFRERE G., 1976. Effets des sucres et des protéines sur l'alcoolémie. Rev. Alc., 22, 73-86.

LOWENSTEIN L. M. SIMONE R., BOUTLER P., NATHAN P., 1970. Effect of fructose on alcohol concentrations in the blood in man. J. amer. Med. Ass., 123, 1899-1901.

LUNDQUIST F., WOLTHERS H., 1958. The influence of fructose on the kinetics of alcohol elimination in man. Acta pharmacol. toxicol., 14, 290-294.

STUHLFAULT K., NEUMAIER H., 1954. Die Wirkung der Laevulose auf Alkoholintoxikationen. Med. Klin., 46, 591-693. 